\title{
Cladocolea loniceroides, un nuevo registro para la flora de Aguascalientes, México
}

\author{
Cladocolea loniceroides, a new record for \\ the flora of Aguascalientes, Mexico
}

Manuel Higinio Sandoval-Orłega *凶, María Elena Siqueiros-Delgado*

Sandoval-Ortega, M. H., \& Siqueiros-Delgado, M. E. (2019). Cladocolea loniceroides, un nuevo registro para la flora de Aguascalientes, México. Investigación y Ciencia de la Universidad Autónoma de Aguascalientes, 27(78), 51-54.

\section{RESUMEN}

La familia Loranthaceae está integrada por 77 géneros y alrededor de 950 especies principalmente hemiparásitas, entre ellas se encuentra Cladocolea loniceroides (Tiegh.) Kuijt que es una especie endémica de México, anteriormente reportada para Colima, Ciudad de México, Guerrero, Jalisco, México, Michoacán, Morelia y Oaxaca. El objetivo del presente trabajo es reportar la presencia de Cladocolea Ioniceroides para Aguascalientes y proporcionar descripciones taxonómicas de género y especie para su identificación. Dentro del estado, C. Ioniceroides se localiza dentro de la ciudad de Aguascalientes parasitando especies ornamentales.

\section{ABSTRAC}

The Loranthaceae family is composed by 77 genera and around 950 species mainly hemiparasitic, among them is found Cladocolea loniceroides (Tiegh.) Kuijt which is a Mexican endemic species previously reported for Colima, Mexico City, Guerrero, Jalisco,

Palabras clave: muérdago; planta parásita; Loranthaceae.

Keywords: mistletoe; parasitic plant; Loranthaceae.

Recibido: 19 de julio de 2018, aceptado: 6 de marzo de 2019

* Herbario HUAA, Departamento de Biología, Centro de Ciencias Básicas, Universidad Autónoma de Aguascalientes. Avenida Universidad 940, Ciudad Universitaria, C. P. 20131, Aguascalientes, Ags., México. Correo electrónico: m.higinio.s@hotmail.com; masiquei@correo.vaa.mx. ORCID: http://orcid.org/0000-0003-1396-9024; http://orcid.org/0000-00020865-3589.

$\triangle$ Autor para correspondencia
Mexico, Michoacan, Morelia and Oaxaca. The objective of the present work is to report the presence of Cladocolea loniceroides for Aguascalientes and to provide taxonomic descriptions of genus and species for their identification. Within the state, C. loniceroides is located in the city of Aguascalientes, parasitizing ornamental species.

\section{INTRODUCCION}

La familia Loranthaceae Juss. está integrada por 77 géneros y alrededor de 950 especies (Stevens, 2001). El género Cladocolea Tiegh. es nativo del nuevo mundo y está integrado por alrededor de 25 especies (Calderón de Rzedowski, 2001), la mayoría de especies se concentra al norte del istmo de Tehuantepec (Kuijt, 1975), unas pocas se distribuyen en Centroamérica y Sudamérica. Del total de especies 19 son nativas de México y 17 son endémicas (Villaseñor, 2016). Son plantas hemiparásitas, y en teoría sólo utilizan a sus hospedantes para extraer agua (AlvaradoRosales \& Saavedra-Romero, 2005).

Cladocolea Ioniceroides (Van Tieghem) Kuijt parasita árboles y arbustos. Su presencia ha sido correlacionada con las condiciones de sitios pobres, específicamente con la alta compactación y baja fertilidad del suelo, factores que favorecen su frecuente aparición (Cid-Villamil \& Bye, 1998) y se le ha reportado parasitando un amplio número de especies de diversas familias a comparación de otras especies del mismo género (Alvarado-Rosales \& Saavedra-Romero, 2005).

El objetivo del presente trabajo es reportar la presencia de C. Ioniceroides en Aguascalientes y 
issn 1665-4412, e-issn 2521-9758

Sandoval-Ortega, M. H., \& Siqueiros-Delgado, M. E.
52

proporcionar descripciones taxonómicas de género y especie para su identificación.

MÉEIODOS

Las colectas se llevaron a cabo en la zona urbana de la ciudad de Aguascalientes durante el mes de junio de 2018. Los ejemplares vegetales fueron colectados siguiendo la metodología propuesta por Engelmann (1986). En el sitio de colecta se tomaron coordenadas geográficas con base en Datum WGS 84. El material colectado fue identificado por medio de claves taxonómicas especializadas (Calderón de Rzedowski, 2001; García-Regalado, 1998; Kuijt, 1975; Vázquez-Collazo, Villa-Rodríguez, \& Madrigal-Huendo, 2006). Los ejemplares colectados fueron depositados en el Herbario de la Universidad Autónoma de Aguascalientes (HUAA) y se enviaron duplicados al Herbario de la Universidad Autónoma del Estado de Morelos (HUMO).

\section{RESULTADOS}

Clave para identificar los géneros de la familia Loranthaceae presentes en Aguascalientes.

1 Flores bisexuales, más de $20 \mathrm{~mm}$ de longitud, tépalos fusionados basalmente, rojos o anaranjados. Psittacanthus

1 Flores generalmente unisexuales, menos de $20 \mathrm{~mm}$ de longitud, tépalos libres, verdosos, blancos o blanco- amarillentos.......................Cladocolea

\section{Cladocolea Tiegh.}

Plantas arbustivas, hemiparásitas, generalmente dioicas, glabras o pubescentes. Tallos numerosos, erectos, volubles o péndulos, teretes o cuadrangulares, superficie lisa, irregular, agrietada o con lenticelas evidentes; comúnmente con raíces adventicias cerca de la base. Hojas sésiles, subsésiles - pecioladas, opuesta o alternas, simples, láminas foliares lanceoladas, ovadas, obovadas, elípticas - lineares, en ocasiones reducidas a escamas, margen entero, ápice agudo a redondeado o mucronado, pinatinervadas. Inflorescencias axilares, pedunculadas, espigas, capítulos, dicasios o racimos, rara vez flores solitarias, brácteas por lo común presentes. Flores generalmente unisexuales, menos de $20 \mathrm{~mm}$ de longitud, sésiles o pediceladas, brácteas presentes, bractéolas ausentes, calículo presente, entero o dentado, perianto de 4-6 tépalos, tépalos libres, verdosos, blancos o blanco-amarillentos, estambres 4-6, dimórficos o monomórficos, filamentos fusionados a los tépalos, anteras de 4 tecas, estilo contorsionado o geniculado, rara vez erecto, estilo capitado o poco diferenciado, ovario globoso a obovoide; nectario presente en forma de anillo alrededor del estilo o estilodio. Fruto baya carnosa de superficie lisa, monospermo.

Género con aproximadamente 25 especies, 19 reportadas para México y sólo una presente en Aguascalientes.

Cladocolea loniceroides (Tiegh.) Kuijt, J. Arnold Arbor. 56(3): 306. 1975. Struthanthus loniceroides (Tiegh.) Engl. Nat. Pflanzenfam. (ed. 2) 16b: 173. 1935. Loxonia loniceroides Tiegh., Bull. Soc. Bot. France 42: 387. 1895. (TIPO: México, J.A. Pavon sn. Isotipo: P) (figura 1).

Struthanthus hunnewellii I.M. Johnt, Contr. Gray Herb. 95: 53. 1931. (TIPO: México, Morelos: on Alnus in Woods north Cuernavaca, Feb. 28 1931, F. W. Hunnewell 11854. Holotipo: $\mathrm{GH})$.

Struthanthus mexicanus Calderón, Cact. Suc. Mex. 17(4): 99-102, 1972. (TIPO: Mexico, Michoacán: Las Manzanillas, 22 Apr 1971, J. Rzedowski 28122. Holotipo: ENCB. Isotipo: MO).

Planta arbustiva, dioica, con pubescencia velutina. Tallos ramificados, rectos o péndulos, de $20-100 \mathrm{~cm}$ o más de largo. Hojas opuestas o alternas, subsésiles o sésiles, peciolo cuando presente no mayor a $2 \mathrm{~mm}$; láminas ovadas o elípticas, de $20-50 \mathrm{~mm}$ de largo por 5-25 mm de ancho, glabrescentes, ápice agudo o mucronado, a veces obtuso, borde entero, base cuneada a obtusa, venación pinnada poco evidente, sólo la vena media prominente en el envés. Inflorescencias pedunculadas, pedúnculos de 2-10(15) mm de largo, capítulos de 3-10 flores, subtendidos por un invólucro de brácteas elípticas a lanceoladas, de 4-5(6) mm de largo y ápice agudo. Flores unisexuales de hasta $5 \mathrm{~mm}$ de longitud, sésiles o subsésiles, las masculinas con calículo alrededor de $1 \mathrm{~mm}$ de largo de borde entero, perianto de seis tépalos blanquecinos de 3-3.5 $\mathrm{mm}$ de largo y 1-1.5(2) mm de ancho, velutinos; flores femeninas con calículo de una sola pieza de borde entero, de menos de $1 \mathrm{~mm}$ de largo, perianto de 6 tépalos estrechamente oblongos de 2-3.5(4) $\mathrm{mm}$ de largo y alrededor de $1 \mathrm{~mm}$ de ancho, velutinos, estilo contorsionado distalmente y de $3-4 \mathrm{~mm}$ de largo, estigma capitado y diminutamente papilado, ovario de hasta $1 \mathrm{~mm}$ de largo, nectario velutino. Fruto rojo oscuro en la madurez, carnoso, elipsoide de (5)6-8 mm de largo por (3)4-5 mm de diámetro, glabro. 


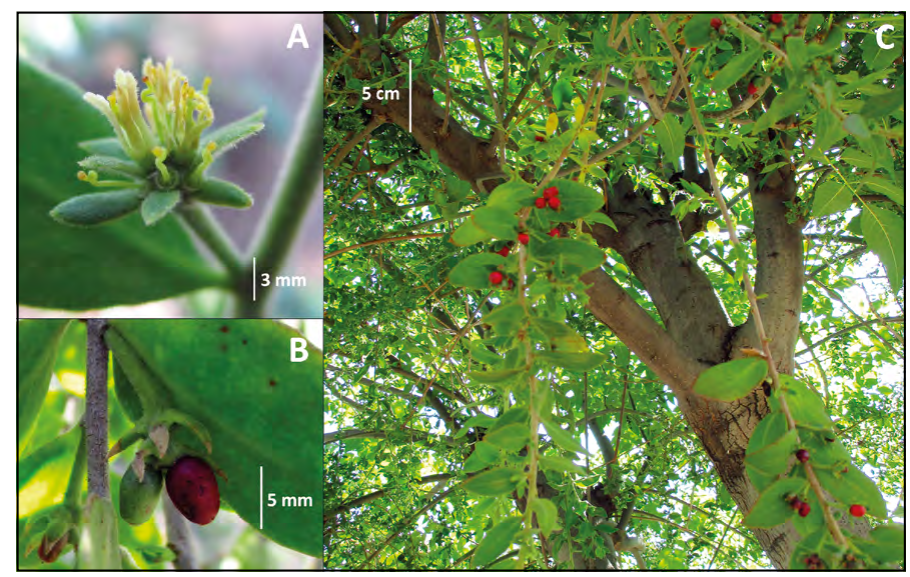

Figura 1. Cladocolea loniceroides. A) Detalle de inflorescencia. B) Detalle de fruto. C) Vista general .

Fotografía del equipo de investigación.

En el estado de Aguascalientes C. Ioniceroides se localiza en el municipio de Aguascalientes, dentro de la zona urbana de la ciudad de Aguascalientes a 1,880 m s. n. m., parasitando especies de álamo (Populus sp.), fresno (Fraxinus sp.) y olmo (Ulmus sp.) (figura 2). Atrae a gran número de abejas cuando se encuentra en flor.

Ejemplares examinados: Aguascalientes: Parque Rodolfo Landeros Gallegos, Sandoval-Ortega 1002 (HUAA, HUMO).

\section{DISCUSIÓN}

Anteriormente para familia Loranthaceae se había reportado solo el género Psittacanthus en el estado de Aguascalientes (García-Regalado, 1998), esto con base en la clasificación más reciente propuesta por el Angiosperm Phylogeny Group, en donde el género Phoradendron Nutt. ya no se incluye en esta familia (Stevens, 2001).

En México, C. Ioniceroides es una especie nativa y endémica, que se había reportado para Colima, Ciudad de México, Guerrero, Jalisco, México, Michoacán, Morelia y Oaxaca (Villaseñor, 2016) y que se reporta por primera vez en Aguascalientes en el presente trabajo. Hasta el momento se ha localizado únicamente en el parque Rodolfo Landeros Gallegos dentro de la ciudad de Aguascalientes, parasitando especies ornamentales, y su presencia se trata muy posiblemente de una introducción. Un caso similar ocurre en Ciudad de México, donde se le ha reportado en Coyoacán parasitando árboles de Acacia, Ligustrum y árboles frutales (Calderón de Rzedowski, 2001) y en la zona chinampera de Xochimilco parasitando una gran cantidad de árboles de varias especies de manera severa (AlvaradoRosales \& Saavedra-Romero, 2005). Su presencia en la Ciudad de México también es considerada una introducción relativamente reciente (Calderón de Rzedowski, 2001).

Pese a que Cladocolea oligantha (Standl. \& Steyerm.) Kuijt. es la especie con más amplia distribución geográfica de todo el género, distribuyéndose en México de Jalisco a Chiapas y en Centro América de Guatemala a Panamá (CházaroBasáñez, Oliva-Rivera, Ramón-Farías, \& VázquezGarcía, 2005), parece ser que C. Loniceroides es la especie mayormente colectada (Kuijt, 1975) y fue además el primer muérdago introducido accidentalmente que ha sido documentado para México. En 1971 se observó sobre árboles de "trueno" (Ligustrum sp.) en Ciudad de México, y se cree que estos árboles fueron llevados de un vivero de Cuernavaca, Morelos (Cházaro-Basáñez et al., 2005).

Las especies de la familia Loranthaceae son una fuente importante de néctar $y$ frutos para las aves, algunas de las cuales se alimentan casi exclusivamente de los frutos de este grupo (Stevens, 2001) y como sucede con los muérdagos en general, las aves son los principales dispersores de semillas (Watson, 2001), dicha dispersión ocurre por medio de tres mecanismos:

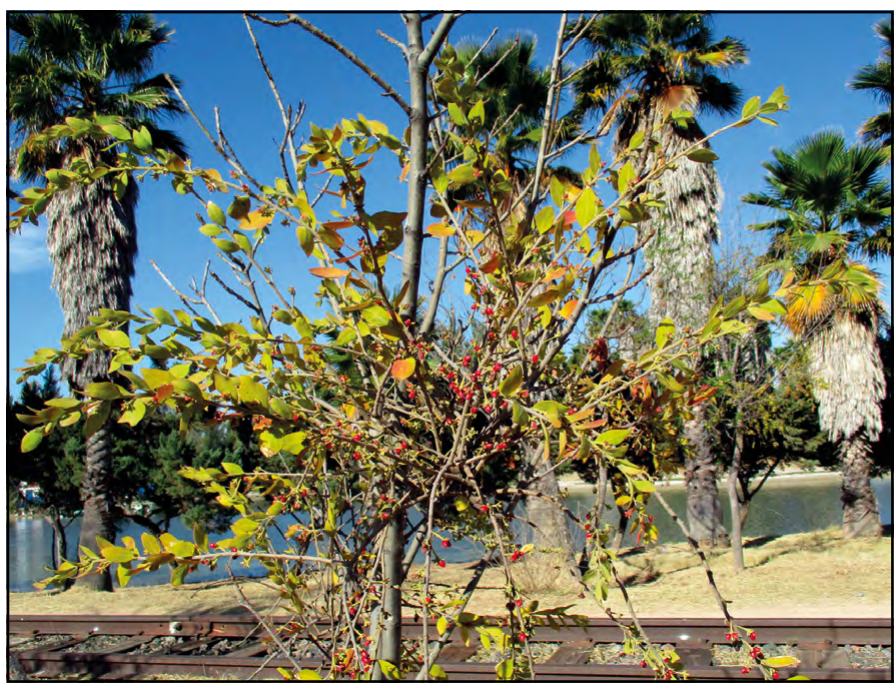

Figura 2. Cladocolea loniceroides parasitando fresno (Fraxinus sp.). Fotografía del equipo de investigación. 
issn 1665-4412, e-issn 2521-9758

Sandoval-Ortega, M. H., \& Siqueiros-Delgado, M. E.
1) La defecación

2) La regurgitación

3 El abandono de las semillas pegajosas sobre las ramas del hospedero.

Se ha adjudicado a las aves migratorias la dispersión a larga distancia de otras especies de muérdagos a través de endozoocoria (CházaroBasáñez \& Oliva-Rivera, 1991). No obstante, es posible que la presencia de $C$. Ioniceroides en Aguascalientes no se deba a la dispersión de semillas por esta vía, como es el caso de otras especies del género Cladocolea según explica Cházaro-Basáñez et al. (2005) y, en cambio, se trate de una introducción causada por el hombre mediante la plantación de árboles infectados, ya que se encuentra parasitando especies ornamentales en un parque dentro de la zona urbana. Sin embargo, es necesario realizar estudios adicionales que permitan esclarecer si su presencia en el estado es a causa del hombre o por vías naturales. Debido a la zona en la que se encuentra actualmente y su forma de dispersión natural, C. Ioniceroides representa una amenaza para áreas verdes adyacentes y la vegetación nativa de Aguascalientes.

CONCLUSIONES

C. Ioniceroides es un nuevo registro tanto de género como de especie para la flora de Aguascalientes, por lo que actualmente en el estado la familia Loranthaceae está representada por dos géneros y un total de tres especies. Debido a que C. Ioniceroides es una planta hemiparásita que puede causar daños severos a su hospedero, representa una amenaza para las áreas verdes de la ciudad de Aguascalientes, otras zonas urbanas y áreas forestales del estado, por lo que es necesario tomar medidas para su control.

\section{REFERENCIAS}

- Alvarado-Rosales, D., \& Saavedra-Romero, L. de L. (2005). El género Cladocolea (Loranthaceae) en México: Muérdago verdadero o injerto. Revista Chapingo. Serie Ciencias Forestales y del Ambiente, 11 (1), 5-9.

- Calderón de Rzedowski, G. (2001). Loranthaceae. En G. Calderón \& J. Rzedowski (Eds.), Flora del Valle de México (2a. ed., p. 106). México: Instituto de Ecología, A.C.-CONABIO.

- Cházaro-Basáñez, M. J., \& Oliva-Rivera, H. (1991). Dendrophthora costaricensis (Loranthaceae), un nuevo registro para la flora de México. Acta Botánica Mexicana, 13, 31-38.

- Cházaro-Basáñez, M. J., Oliva-Rivera, H., Ramón-Farías, F., \& Vázquez-García, J. A. (2005). Cladocolea oligantha (Loranthaceae) un nuevo registro para Veracruz, México, y datos generales sobre este taxón. Polibotánica, 20, 1-15.

- Cid-Villamil, R. M., \& Bye, R. A. (1998). Site conditions of an urban wooded area of Mexico City that hosts Cladocolea Ioniceroides Van Tieghem (Loranthaceae). Selbyana, 19(2), A 272.

- Engelmann, G. (1986). Instructions for the collection and preservation of botanical specimens. Annals of the Missouri Botanical Garden, 73(3), 504-507.
- García-Regalado, G. (1998). La familia Loranthaceae (Injertos) del estado de Aguascalientes, México. Polibotánica, 7(mayo), $1-14$.

- Kuijt, J. (1975). The genus Cladocolea (Loranthaceae). Journal of the Arnold Arboretum, 56(3), 265-335.

- Stevens, P. F. (2001). Angiosperm Phylogeny Website [Portal electrónico]. Recuperado el 14 de julio de 2018, de http:// www.mobot.org/MOBOT/research/APweb/

- Vázquez-Collazo, I., Villa-Rodríguez, A., \& Madrigal-Huendo, S. (2006). Los muérdagos (Loranthaceae) en Michoacán. Libro Técnico Núm. 2. Michoacán, México: SAGARPA-INIFAP.

- Villaseñor, J. L. (2016). Checklist of the native vascular plants of Mexico. Revista Mexicana de Biodiversidad, 87 (3), 559-902. doi: 10.1016/j.rmb.2016.06.017

- Watson, D. M. (2001). Mistletoe-A keystone resource in forests and woodlands worldwide. Annual Review of Ecology and Systematics, 32, 219-249. doi: 10.1146/annurev. ecolsys.32.081501.114024 